\title{
Effect of oral administration of lactic acid bacteria on colony performance and gut microbiota in indoor-reared bumblebees (Bombus terrestris)
}

\author{
Annelies Billiet ${ }^{1}$, Ivan Meeus ${ }^{1}$, Margo CnOCKaerT $^{2}$, Peter Vandamme ${ }^{2}$, \\ Annette Van OystaeYen ${ }^{3}$, Felix Wäckers ${ }^{3}$, Guy SMAGGHE ${ }^{1}$ \\ ${ }^{1}$ Laboratory of Agrozoology, Department of Crop Protection, Faculty of Bioscience Engineering, Ghent University, \\ Coupure Links 653, 9000, Ghent, Belgium \\ ${ }^{2}$ Laboratory of Microbiology, Department of Biochemistry and Microbiology, Faculty of Sciences, Ghent University, K. \\ L. Ledeganckstraat 35, 9000, Ghent, Belgium \\ ${ }^{3}$ Biobest Belgium NV, Ilse Velden 18, 2260, Westerlo, Belgium
}

Received 9 December 2015 - Revised 25 March 2016 - Accepted 7 April 2016

\begin{abstract}
In this study, we investigated if oral administration of lactic acid bacteria could increase the colony performance of reared bumblebees. We found that a continuous administration of Lactobacillus kunkeei LMG 18925 and Lactobacillus crispatus LMG 9479 could partly compensate the effects of low nutritional pollen, but a permanent colonization in the gut was not detected with Illumina MiSeq 16S rRNA sequencing. Contrary to expectations, a single administration of $L$. kunkeei LMG 18925 to a high nutritional pollen led to a lower total drone mass. A Bombus -specific strain, Bifidobacterium actinocoloniiforme R-53049, showed potential to colonize the gut permanently after three administrations. Our study represents a first screening for the potential use of probiotic strains in bumblebees. We conclude that both diet and host specificity of bacteria might have an effect on colony performance of indoor-reared bumblebees and play a role in the gut colonization success.
\end{abstract}

\section{Bifidobacterium / Lactobacillus / probiotics / Bombus terrestris / colony development / gut microbiota}

\section{INTRODUCTION}

Many endogenous bacteria have a mutualistic relationship with their insect host and play a role in digestion, nutrient production, and pathogen protection (Koch and Schmid-Hempel 2012; Engel and Moran 2013; Cariveau et al. 2014). Social insects like honeybees (Apis) and bumblebees (Bombus) harbor a distinct gut microbiota

Electronic supplementary material The online version of this article (doi:10.1007/s13592-016-0447-5) contains supplementary material, which is available to authorized users.

Corresponding author: G. Smagghe,

Guy.Smagghe@ugent.be

Handling Editor: Klaus Hartfelder
(Martinson et al. 2011). This gut microbiota probably contributes to the success of the colony, as dysbiosis of the microbiota has been associated with a reduced health in bees (Sabree et al. 2012; Vasquez et al. 2012).

Commercially reared honeybees and bumblebees have a high economical value as crop pollinators (Losey and Vaughan 2006; Velthuis and van Doorn 2006). Some of the rearing techniques used in the production of honeybees and bumblebees can have a detrimental effect on the bacterial community in the gut. For example, in the USA, domesticated honeybees are often treated with antibiotics to control foulbrood infections (Tian et al. 2012), but this is also impairing the gut bacteria. The mass rearing of bumblebees typically takes place in closed facilities, to avoid disease contamination, but at the same time, it can also 
result in a reduction of the bacterial diversity in the bumblebee gut (Meeus et al. 2015). Gaining further insight into the functionality of the bee gut microbiota holds promising opportunities to enhance the fitness of the colony. In humans and several animals, strains of Lactobacillus and Bifidobacterium are already widely used in probiotic formulations. In mammals and fish hatcheries, for example, the use of probiotic strains has increased growth and reduced diseases without the use of antibiotics (Kawakami et al. 2010; Magnadottir 2010; Bovera et al. 2012; Piccolo et al. 2015). Also in insects, the administration of probiotic bacterial strains can improve survival, body size, growth rate, mating, and fecundity, which was demonstrated in fruit flies, silkworms, moths, and olive flies (Niyazi et al. 2004; Masthan et al. 2010; Gavriel et al. 2011; Sacchetti et al. 2014). Several studies on honeybees have shown that lactobacilli can provide protection against Paenibacillus larvae, the bacterium causing American foulbrood disease, and Melissococcus plutonius, the bacterium causing European foulbrood disease, as well as reduced mortality of infected larvae (Evans and Lopez 2004; Forsgren et al. 2010; Wu et al. 2014). Furthermore, different studies have shown that the administration of commercially available probiotic formulations in honeybees decreased mortality, increased body mass, increased fat body and the size of the faucal gland, and increased the merocrin-type secretion and quantities of peritrophic membranes, thus providing a better digestion (Kaznowski et al. 2005; KazimierczakBaryczko and Szymas 2006; Szymas et al. 2012). In stingless bees, Bacillus can play an important role by secreting enzymes that can soften the pollen wall and cause fermentation, contributing to digestion (Gilliam et al. 1985, 1990).

As several positive effects on honeybees have been demonstrated in previous experiments, we wanted to investigate the effect of the administration of Lactobacillus and Bifidobacterium strains to indoor-reared bumblebees in three experiments. We used bumblebee microcolonies to assess their performance based on parameters such as colony development and drone production. We also investigated by use of Illumina MiSeq sequencing, whether we could detect colonization of the supplemented bacteria and whether there was an effect on the microbial gut community. In a first experiment, we tested if a continuous supplementation of several strains of Lactobacillus and Bifidobacterium could compensate the effects of low nutritional pollen. In a second experiment, we investigated if Lactobacillus kunkeei LMG 18925 would also be able to increase the reproduction, when supplied only once and high nutritional pollen was provided. In a third experiment, we provided bumblebee microcolonies at three different points in time, with Bifidobacterium actinocoloniiforme R-53049, isolated from the gut of a wild bumblebee. The objective here was to realize a better colonization, as we expected that a bumblebee-specific bacterium is better adapted to the bumblebee gut than a non-host bacterium.

\section{MATERIALS AND METHODS}

\subsection{Bumblebees and their developmental parameters in microcolonies}

In all bioassays, we used microcolonies consisting of five newly emerged Bombus terrestris workers. The bumblebees were obtained from an indoor massproduction facility (Biobest, Westerlo, Belgium). Microcolonies have been proven comparable to queen-right colonies for investigating microbiota (Meeus et al. 2013), and microcolonies follow a welldefined development pattern with quantifiable parameters, such as time point of egg laying, larval development time, pupation time, and reproductive output. In each microcolony, one worker becomes dominant and starts laying unfertilized eggs that develop into drones, while the other workers take care of the brood. Several developmental parameters were assessed daily, during 50 days, in 10 microcolonies for each treatment: the number of days until first egg, first pupa, first drone emergence, the number of drones, the total drone mass per microcolony, and the average mass per drone. The microcolonies were kept under standardized laboratory conditions at $30{ }^{\circ} \mathrm{C}$ and continuous darkness during the experiments. All microcolonies were provided with Biogluc ${ }^{\circledR}$ sugar syrup ad libitum. The pollen and the 
bacterial treatments are described below for each experiment. All pollen was $15 \mathrm{kGy}$ radiation sterilized.

\subsection{Cultivation of bacterial strains}

Lactobacillus acidophilus LMG 11430, Lactobacillus crispatus LMG 9479, Lactobacillus kunkeei LMG 18925, Bifidobacterium asteroides LMG 10735 and LMG 11581, Bifidobacterium coryneforme LMG 19811, and Bifidobacterium actinocoloniiforme R53049 were cultured on selective agar plates (Supplementary Table S1). All bacterial strains were obtained from the Belgian Coordinated Collections of Microorganisms at the Laboratory of Microbiology of Ghent University (BCCM/LMG), except $B$. actinocoloniiforme R53049 which was isolated and identified from a bumblebee gut in the lab. All bacteria were cultured at $37^{\circ} \mathrm{C}$ in anaerobic conditions, except L. kunkeei LMG 18925 which was aerobically cultured at $28{ }^{\circ} \mathrm{C}$. Bacterial colonies were picked up from their agar plates 2 to 3 days after inoculation and cells were suspended into physiological saline. This bacterial suspension was then added to the pollen provided to the bumblebee microcolonies.

\subsection{Illumina MiSeq sequencing}

The composition in the gut microbiota was assessed by dissection of the mid- and hindgut, using disinfected dissection material. The gut was subsequently crushed in a $170-\mu \mathrm{L}$ lysozyme solution $(100 \mathrm{mg} / \mathrm{mL})$ and DNA extraction was performed as described in Meeus et al. (2013). The hypervariable V4 region (254 bp) of the 16S rRNA was amplified in triplicate, using the $515 \mathrm{~F}$ and $806 \mathrm{R}$ primers designed by Caporaso et al. (2011). Sample preparation and Illumina sequencing were performed as described in Billiet et al. (2015). Sequences derived from the Illumina Miseq sequencing were analyzed with the mothur software v. 1.31.1 (Schloss et al. 2009), mainly following the standard operating procedure available on http:/www.mothur.org/wiki/ MiSeq_SOP, date December 2013. The raw data are publicly available on NCBI's Sequence Read Archive (SRA) under accession number SRP065023. The analysis of the Illumina data was performed as described in Billiet et al. (2015). The reads of the samples were calculated in percentages, expressing the relative abundance of each OTU. In this analysis, we chose to retain the OTUs that were represented by more than $0.05 \%$ of the reads. Community richness was calculated with the Chao1 estimator (alpha diversity) and community diversity with the Shannon index (beta diversity). The bacterial evenness (e) was calculated as $e=H / \ln S$, where $H$ is the Shannon index and $S$ is the number of OTUs.

\subsection{Experimental setups}

\subsubsection{Continuous supplementation of Lactobacillus and Bifidobacterium strains to low nutritional pollen}

In this experiment, we investigated the effects of six bacterial strains: L. acidophilus LMG 11430, L. crispatus LMG 9479, L. kunkeei LMG 18925, B. coryneforme LMG 19811, B. asteroides LMG 10735 and $B$. asteroides LMG 11581. These bacterial species were identified in the honeybee gut (Forsgren et al. 2010; Audisio et al. 2011). The strains were continuously administered in the pollen mixture which was replaced every 2 or 3 days with a freshly prepared pollen mixture. All microcolonies were fed with low nutritional pollen consisting of a honeybee-collected pollen mixture, further referred as pollen A. The pollen mixture consisted of pollen $(90.32 \% \mathrm{~m} / \mathrm{m})$, sugar syrup $(6.45 \% \mathrm{~m} / \mathrm{m})$, and one bacterial strain suspended in physiological saline $(3.23 \% \mathrm{~m} / \mathrm{m})$. The number of colony-forming units (CFU) per gram pollen for each treatment is shown in Table I. The 10 microcolonies in the control group received the same pollen mixture with pollen $(90.32 \% \mathrm{~m} / \mathrm{m})$, sugar syrup $(6.45 \% \mathrm{~m} / \mathrm{m})$, and physiological saline $(3.23 \% \mathrm{~m} / \mathrm{m})$, without bacterial supplementation.

Each bacterial strain was supplied to 10 microcolonies from the start of the experiment (day 0 ) until day 42 . From day 42 to day 50 , all microcolonies received pollen without bacterial supplementation. We examined the gut microbial composition of the bumblebee workers of the control treatment, L. kunkeei LMG 18925 treatment and L. crispatus LMG 9479 treatment by use of Illumina sequencing. We therefore sampled one bumblebee worker of five to six microcolonies of each of these treatments at day 44. At this point, we expected the bacterial strain to be present in the digestive tract of the bumblebees. At day 50, we sampled again one bumblebee worker of the same microcolonies of these treatments. Detection of the bacterial strain at 
Table I. The identification details, the biological origin, and the number of colony-forming units (CFU) per gram pollen of the six bacterial strains used in the first experiment.

Bacterial species Strain number Biological origin

Colony-forming units per gram pollen (CFU/g pollen)

\begin{tabular}{llll}
\hline Lactobacillus crispatus $^{\text {a }}$ & LMG 9479 & Eye & $3.23 \times 10^{3}$ \\
Lactobacillus kunkeei $^{\text {a }}$ & LMG 18925 & Partially fermented grape juice & $3.55 \times 10^{4}$ \\
Lactobacillus acidophilus $^{\text {a }}$ & LMG 11430 & Human & $1.00 \times 10^{5}$ \\
Bifidobacterium asteroides $_{\text {Bifidobacterium asteroides }}$ & LMG 10735 & Honeybee, hindgut & $1.19 \times 10^{7}$ \\
Bifidobacterium coryneforme & LMG 11581 & Honeybee, hindgut & $1.29 \times 10^{7}$ \\
\hline
\end{tabular}

${ }^{\text {a }}$ Bacterial species was $100 \%$ identical with the species isolated from the honeybee gut, but was isolated from another environment

this stage would point toward a potential colonization of the strain in the bumblebee gut.

Statistical differences in the days until first egg, first pupa, and first drone were analyzed using the Kruskal-Wallis test. Statistical differences in the number of drones, the drone mass per microcolony, and the mass per drone were analyzed using ANOVA with two-sided Dunnett's post hoc test. This test allows comparison of multiple treatment groups to one control group. The critical value was $P=0.050$.

\subsubsection{Fitness effects of a single supplementation of L. kunkeei LMG 18925 to two high nutritional pollen types}

We tested the effect of a single supplementation of $L$. kunkeei LMG 18925 to two types of high nutritional pollen, which will be further referred to as pollen $\mathrm{B}$ and pollen C. Hence, we created 4 groups of 10 microcolonies each: a treatment group and a control group for both pollen types.

Pollen mixtures were prepared, as described in the previous bio-assay, with $3.55 \times 10^{4} \mathrm{CFU}$ of $L$. kunkeei LMG 18925 per gram pollen mixture. The control treatment received the same pollen mixture, but without bacterial administration. After the first week, a freshly prepared pollen mixture was provided ad libitum without bacterial supplementation and replaced weekly. Statistical differences in the days until first egg, first pupa, and first drone were analyzed using the Mann-Whitney $U$ test. Statistical differences in the number of drones, the drone mass per microcolony, and the mass per drone were analyzed using a two-sided $t$ test.

\subsubsection{Three supplementations of bumblebee isolate B. actinocoloniiforme R-53049}

In a third experiment, we tested the effect of administration with $B$. actinocoloniiforme R53049. This strain was isolated from the gut of Bombus pascuorum that was sampled in Belgium, and showed a $100 \%$ (434/434 bp) similarity with B. actinocoloniiforme strain DSM $22766^{\mathrm{T}}$ (data not shown). The bacterium was supplemented to the food by spraying a bacterial suspension on the pollen at three points of time: on day 0 , day 14 , and a last time at day 28. Each microcolony was provided with $0.5 \mathrm{~mL}$ physiological saline $(0.86 \% \mathrm{NaCl})$ in which $1.76 \times 10^{9} \mathrm{CFU}$ of $B$. actinocoloniiforme R-53049 was suspended. In the control treatment, $0.5 \mathrm{~mL}$ of physiological saline was sprayed on the pollen by use of a spraying device. To ensure low nutritional conditions, the microcolonies were fed a pollen mixture consisting of $30 \%$ pollen and $70 \%$ of a pollen substitute.

The days until first egg, first pupa, and first drone between the B. actinocoloniiforme R-53049 treatment and the control group were analyzed using the MannWhitney $U$ test. Differences in the number of drones, drone mass per microcolony, and mass per drone were 
analyzed using a two-sided $t$ test. The critical value was $P=0.050$.

At day 50, we sampled six bumblebees, originating from three microcolonies to investigate the gut microbiota.

\section{RESULTS}

\subsection{Continuous supplementation of several Lactobacillus and Bifidobacterium strains to low nutritional pollen}

\subsubsection{Assessment of developmental parameters in microcolonies}

In the first experiment, we used pollen A for which the control group produced an average of $18.2 \pm 2.80$ drones per microcolony, a total drone mass per microcolony of $5.27 \pm 0.44 \mathrm{~g}$, and an average mass per drone of 290.2 $\pm 2.6 \mathrm{mg}$.

The Kruskal-Wallis test showed no significant differences between treatments in the number of days until first eggs $(P=0.235)$, days until first pupa $(P=0.870)$, and days until first drone emergence $(P=0.574)$ (supplementary information Table S2). An overall ANOVA test showed no significant effects on the total drone mass per microcolony $(P=0.161 ; F=1.603)$. However, significant differences could be shown for the number of drones $(P=0.020 ; F=2.734)$ and drone mass per microcolony $(P=0.004$; $F=3.707)$. The two-sided Dunnett's post hoc test revealed that the continuous treatment with L. crispatus LMG 9479 resulted in a higher number of drones per microcolony $(28.1 \pm 1.24$ drones; $P=0.056)$ than the control treatment, with a similar mass per drone $(289.8 \pm 6.9 \mathrm{mg} ; P=1.000)$. Compared to the control treatment, also, the continuous supplementation of L. kunkeei LMG 18925 resulted in a trend toward a higher drone production $(27.1 \pm 3.05$ drones; $P=0.090$ ), with a similar mass per drone $(286.0 \pm 5.0 \mathrm{mg} ; P=0.998)$. The other bacterial treatments showed no significant differences when compared to the control group.

\subsubsection{Gut microbiota in samples of treatments L. kunkeei LMG 18925, L. crispatus LMG 9479, and control}

As a continuous administration of L. kunkeei LMG 18925 and L. crispatus LMG 9479 showed some positive effects on reproduction, we investigated the microbial gut composition for these two treatments and the control group, using Illumina Miseq sequencing.

Taxonomic identification of the OTUs and their closest match in GenBank or EzBioCloud are presented in the supplementary information Table S3. The genetic distance of an OTU with its closest bacterial family members is shown for Lactobacillaceae (supplementary information Figure S1) and for the Bifidobacteriaceae (supplementary information Figure S1), based on the 254-bp sequence.

L. kunkeei was detected in very low relative abundances in some samples of all three treatments (Figure 1a, b). Therefore, we cannot conclude whether the administration of L. kunkeei LMG 18925 contributed to colonize the insect gut. L. crispatus was detected in only 1 of the 11 samples; this was in the L. crispatus LMG 9479 treatment in a sample taken at day 44. The overall relative abundance of Lactobacillaceae and Bifidobacteriaceae was not affected by the administration of L. kunkeei LMG 18925 or $L$. crispatus LMG 9479 (Figure 1c, d), and the treatments did not induce major impacts on the community richness (Chao1 estimator), the community diversity (Shannon index), nor evenness (Figure 1e, f).

\subsection{Fitness effects of a single supplementation of $L$. kunkeei LMG 18925 to two high nutritional pollen types}

The control treatment with high nutritional pollen $B$ produced $44.1 \pm 4.17$ drones per microcolony in 50 days, with a total drone mass per microcolony of $13.13 \pm 0.84 \mathrm{~g}$ and an average mass per drone of $283.2 \pm 14.8 \mathrm{mg}$. The single supplementation of L. kunkeei LMG 18925 to pollen B did not result in a significant faster colony development, screened by days until first 
Samples taken at day 44
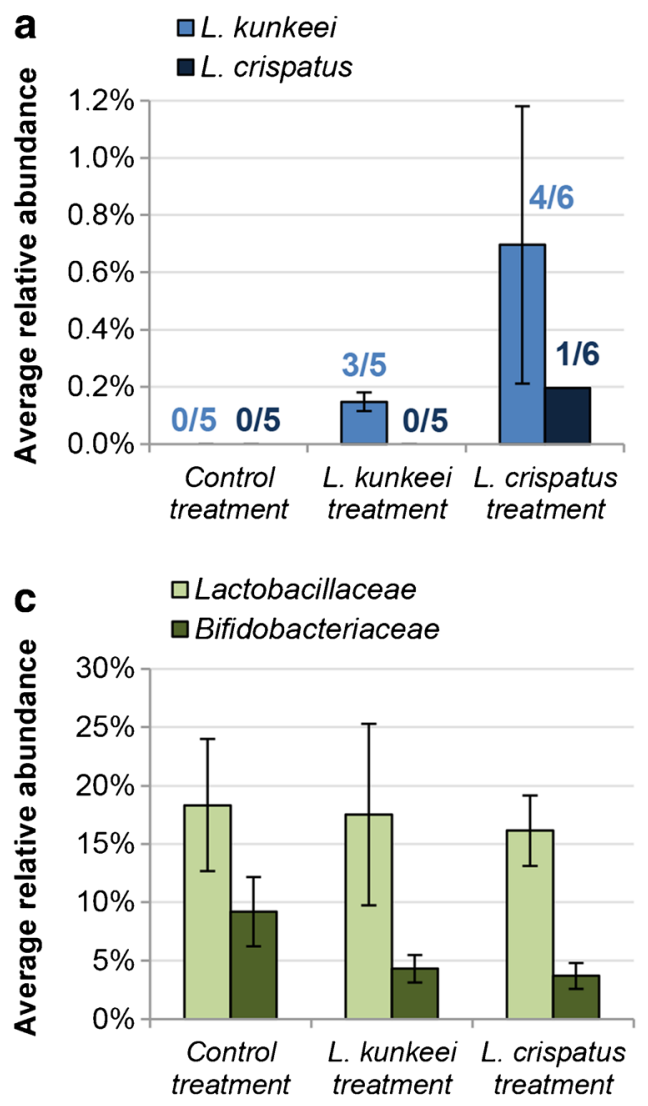

e םChao1 םShannon םEvenness

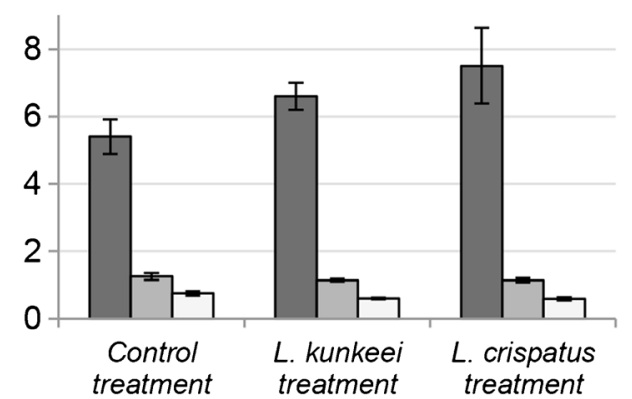

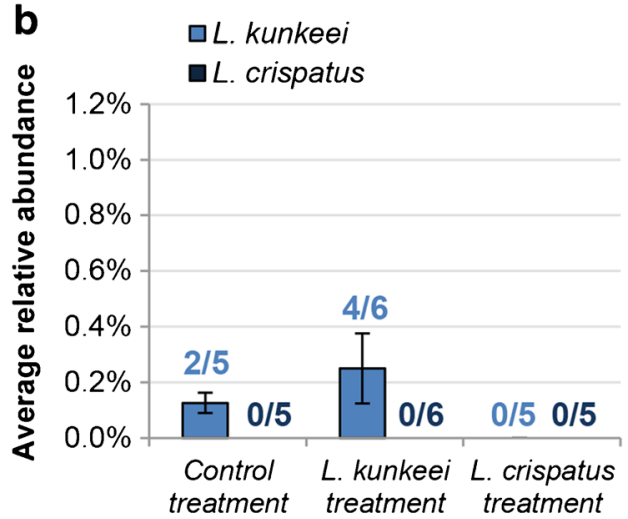

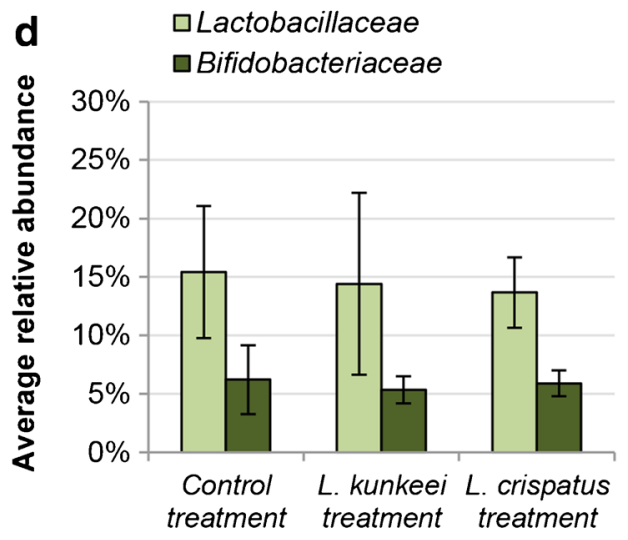

f

$\square$ Chao1 $\square$ Shannon $\square$ Evenness

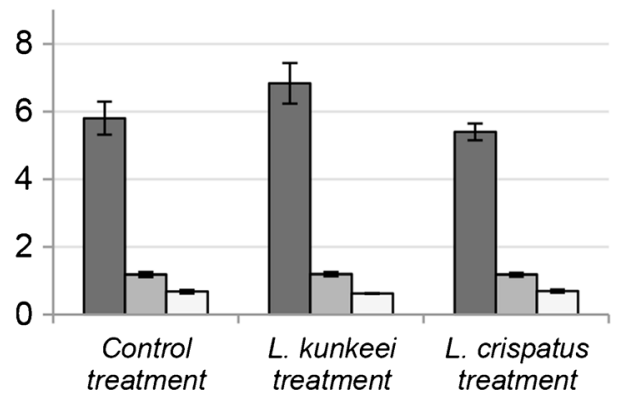

Figure 1. The left column (a, c, e) shows the results of the samples taken at day 44, and the right column (b, d, f) represents the samples taken at day 50 of the control group, the treatments with $L$. kunkeei LMG 18925 and $L$. crispatus LMG 9479. Error bars represent the standard error. a, b The charts represent the average relative abundance of $L$. kunkeei and L. crispatus in the samples in which the bacterium was present. The numbers above the graph represent the prevalence of this sequence in the corresponding treatment. $\mathbf{c}, \mathbf{d}$ The chart shows the relative abundance of all Lactobacillaceae and all Bifidobacteriaceae. e, f The average community richness (Chaol estimator), community diversity (Shannon index), and evenness for each treatment. 
eggs $(P=0.125 ; Z=-1.534)$, first pupa $(P=0.741 ; Z=-0.331)$, and first drone emergence $(P=0.600 ; Z=-0.524)$ (supplementary information Table S2). Compared to the control treatment, microcolonies that were supplemented with L. kunkeei LMG 18925 showed a trend toward a lower drone production per microcolony $(33.8 \pm 3.04$ drones; $P=0.067)$ with a similar mass per drone $(285.6 \pm 6.9 \mathrm{mg} ; P=0.890)$, resulting in a significant lower total drone mass per microcolony $(9.54 \pm 0.75 \mathrm{~g} ; P=0.006)$.

The control group fed with pollen $\mathrm{C}$ produced $27.9 \pm 2.00$ drones per microcolony in 50 days, a total drone mass per microcolony of $8.95 \pm 0.55 \mathrm{~g}$, and a mass per drone of $323.8 \pm 8.9 \mathrm{mg}$. The supplementation of L. kunkeei LMG 18925 to pollen $\mathrm{C}$ did not induce significant differences in days until first egg $(P=0.503 ; Z=-0.669)$, days until first pupa $(P=0.841 ; Z=-0.200)$, and days until first drone $(P=0.412 ; Z=-0.820)$. The number of drones per microcolony was higher $(32.9 \pm 3.50)$ in the control group, but this effect was not significant $(P=0.234)$. The total drone mass was also similar $(9.56 \pm 1.01 \mathrm{~g} ; P=0.603)$. The slightly higher number of drones, but the similar total drone mass, resulted in a significantly lower mass per drone $(290.9 \pm 5.3 \mathrm{mg} ; P=0.005)$, compared to that in the control group fed with pollen $\mathrm{C}$.

\subsection{Three supplementations of bumblebee isolate $B$. actinocoloniiforme R-53049}

\subsubsection{Assessment of developmental parameters in microcolonies}

The control group produced an average number of drones of $17.7 \pm 1.08$ with an average drone mass of $323.5 \pm 9.7 \mathrm{mg}$. Supplementation of $B$. actinocoloniiforme R-53049 to the pollen showed a faster development in days until first egg $(P=0.029 ; Z=-2.189)$ and a slightly faster, but not significant, development in days until first pupa $(P=0.067 ; Z=-1.831)$, compared to the control treatment (supplementary information Table S1). There was, however, no significant effect on time of first drone emergence $(P=0.914 ; Z=-0.108)$, and it also did not impact the number of drones $(18.7 \pm 2.28$ drones;
$P=0.696)$ nor the mass per drone (325.6 $\pm 4.0 \mathrm{mg} ; P=0.843)$.

\subsubsection{Gut microbial composition}

B. actinocoloniiforme was never detected in the samples of the control group $(0.00 \pm 0.00 \%)$, while it was present in four out of six sampled bumblebees of the treatment group. The samples that were positive for B. actinocoloniiforme had an average relative abundance of $2.09 \pm 1.49 \%$. The overall relative abundances of Lactobacillaceae (L. bombi, L. bombicola, L. apis, L. kunkeei) or Bifidobacteriaceae (B. commune, $B$. coagulans ) were not significantly influenced by the $B$. actinocoloniiforme R-53049 treatment. No major changes were observed in the community richness, community diversity, and evenness.

\section{DISCUSSION}

In this study, we demonstrated that a continuous administration of L. kunkeei LMG 18925 and L. crispatus LMG 9479 can positively affect the drone production, when providing low nutritional pollen. It remains unclear which mechanisms are responsible for the increased drone production. Possibly, the supplemented bacterial strains were able to aid in nutrient production. It seems likely that certain bacterial products can benefit bee health, as it was recently demonstrated that metabolites produced by Lactobacillus johnsonii CRL 1647 can increase the honeybee colony fitness (Maggi et al. 2013). Besides nutrient production, bacteria can also help in the digestion of the pollen grain. A thick pollen wall surrounds the inner nutrient-rich protoplasm (Roulston and Cane 2000). Bacteria can produce digestive enzymes softening the pollen wall and helping to release the nutrients from the pollen grain (Engel et al. 2012), and in this way are able to release more proteins needed for reproduction and ovary development (Hoover et al. 2006) or release more amino acids which have been proven to have an effect on the reproduction and growth rate of bumblebee larvae as well (Moerman et al. 2016). We could, however, not demonstrate a similar positive effect when L. kunkeei LMG 18925 
was administered only once to high nutritional pollen $\mathrm{B}$, as it even led to a decrease in drone production. A possible explanation for these findings could be that the effect of microorganisms on their host could be dependent on environmental variables, such as dietary composition. In the case of nutritionally optimal pollen, bumblebees might not need supplementary nutrients provided by the bacteria nor their help in digestion and L. kunkeei LMG 18925 might consume more nutrients from the pollen than it produces for the host. Only for the digestion of low nutritional pollen, which could be either difficult to digest or lacking certain nutrients, bumblebees seemed to benefit from the bacterial administration. The principle of contextdependent benefits of bacteria has been described in aphids: when a parasitoid wasp deposits an egg inside its aphid host, the developing parasitoid wasp larva ultimately kills the host (Oliver et al. 2003, 2008). The secondary symbiont Hamiltonella defensa is able to inhibit the parasitoid development and protects the aphid. Although the aphid does not get killed and thus benefits from the bacterial presence, the protection of $H$. defensa is not free of costs to the aphid, as $H$. defensa consumes many nutrients from the aphid. Aphids carrying $H$. defensa have reduced fecundity and are outcompeted by aphids that do not carry the costly secondary symbiont in environments where no parasitoids are present (Vorburger and Gouskov 2011). Thus, in optimal conditions, the bacterium has a negative effect on the host, while in unfavorable conditions, it is beneficial for the host.

Even though we found positive effects in the continuous administration of L. kunkeei LMG 18925 and L. crispatus LMG 9479, we could not confirm their presence in the gut. For the microbial analysis, we only sampled the midand hindgut. It remains possible that these bacteria did colonize other parts of the gut, such as the crop. In honeybees, it has been shown that the crop harbors a rich diversity of lactic acid bacteria which are tightly attached to the crop wall in a biofilm layer (Olofsson and Vasquez 2008; Olofsson et al. 2014). For future investigations, we propose to investigate the crop, midgut, and hindgut separately, as some bacteria might be restricted to a certain region in the digestive tract.
Host specificity might also play a role in the colonization success of a bacterium. Earlier studies in Apis and Bombus demonstrated that native strains of Snodgrassella showed higher levels of colonization, than non-host Snodgrassella strains (Kwong et al. 2014), which points toward a certain degree of host specificity between microbes and their host. Most of the bacteria administered in our experiments were not Bombus specific, with the exception of $B$. actinocoloniiforme R53049 which was isolated from the gut of a wild $B$. pascuorum and which was previously also found in the guts of wild $B$. terrestris (Meeus et al. 2015). Our experiments confirmed that $B$. actinocoloniiforme was not present in the gut of the reared bumblebees of the control treatment, but three administrations of this bacterium seemed sufficient to colonize the gut of the majority of the sampled bumblebees. Regarding administration frequency, possibly, a few administrations will be sufficient to colonize the gut when applying a host-specific bacterium.

Although B. actinocoloniiforme R-53049 was not able to improve microcolony performance, it is still worthwhile to look further into the effects of the gut bacteria and expand the parameters that we assessed. One especially interesting parameter would be immunity. In our experiments, all colonies were kept under optimal conditions and did not come into contact with potential pathogens. In previous studies with honeybees, beneficial effects of the presence of certain lactic acid bacteria on immunity could be demonstrated (Koch and SchmidHempel 2011). When bumblebees are placed outside in the field and encounter bee diseases from wild bees, the reared bumblebees should be as immunocompetent as possible and the introduction of host-specific bacteria might be helpful.

We can conclude that our study represents a first screening of the potential of probiotic strains in bumblebees and the possible effects for bumblebee rearing. We identified two bacteria, L. kunkeei LMG 18925 and L. crispatus LMG 9479, showing potential to improve colony performance, but we could not demonstrate their ability to colonize the gut. Further research is needed to identify the underlying mechanisms of their beneficial effect. We also demonstrated that $B$. actinocoloniiforme R53049 could effectively colonize the bumblebee 
gut, but this bacterium did not improve colony performance under laboratory conditions. Further research could expand this study to field conditions to assess possible effects on immunity. At this point, there is still not enough knowledge on the functions of the bacteria in the bumblebee gut. In order to develop probiotics for bumblebees, more research should be done with other bacterial species, the combination of several bacteria or prebiotics, and the application methods like the administration frequency.

\section{ACKNOWLEDGMENTS}

We would like to thank Jessy Praet (Laboratory of Microbiology, Department of Biochemistry and Microbiology, Ghent University) for the isolation and identification of Bifidobacterium actincoloniiforme R-53049.

This study was supported by the Institute for the Promotion of Innovation by Science and Technology in Flanders (IWT, Brussels, Belgium) and the Fund for Scientific research (FWO-Flanders, Brussels, Belgium).

Effet de bactéries d'acide lactique, administrées par voie orale, sur la performance de la colonie et le microbiote intestinal chez des bourdons élevés en intérieur (Bombus terrestris)

Bifidobacterium / Lactobacillus / probiotiques / Apidae / développement de la colonie / flore intestinale

Auswirkung der oralen Zuführung von Milchsäurebakterien auf die Kolonieentwicklung von in Innenräumen gehaltenen Hummeln (Bombus terrestris)

Bifidobacterium / Lactobacillus / Probiotika / Bombus terrestris / Kolonieentwicklung / Darmmikrobiota

\section{REFERENCES}

Audisio, M.C., Torres, M.J., Sabate, D.C., Ibarguren, C., Apella, M.C. (2011) Properties of different lactic acid bacteria isolated from Apis mellifera L. bee-gut. Microbiol Res 166(1), 1-13

Billiet, A., Meeus, I., Van Nieuwerburgh, F., Deforce, D., Wäckers, F., Smagghe, G. (2015) Impact of sugar syrup and pollen diet on the bacterial diversity in the gut of indoor-reared bumblebees
(Bombus terrestris). Apidologie DOI . doi:10.1007/s13592-015-0399-1

Bovera, F., Iannaccone, F., Mastellone, V., Nizza, S., Lestingi, A., et al. (2012) Effect of spray application of Lactobacillus plantarum on in vivo performance, caecal fermentations and haematological traits of suckling rabbits. Ital. J. Anim. Sci. 11 (2), e27

Caporaso, J.G., Lauber, C.L., Walters, W.A., Berg-Lyons, D., Lozupone, C.A., Turnbaugh, P.J., Fierer, N., Knight, R. (2011) Global patterns of $16 \mathrm{~S}$ rRNA diversity at a depth of millions of sequences per sample. Proc. Natl. Acad. Sci U.S.A. 108 (suppl. 1), 4516-4522

Cariveau, D.P., Elijah Powell, J., Koch, H., Winfree, R., Moran, N.A. (2014) Variation in gut microbial communities and its association with pathogen infection in wild bumble bees (Bombus ). ISME J. 8, 2369-2379

Engel, P., Moran, N.A. (2013) The gut microbiota of insects - diversity in structure and function. FEMS Microbiol Rev. 37 (5), 699-735

Engel, P., Martinson, V.G., Moran, N.A. (2012) Functional diversity within the simple gut microbiota of the honey bee. Proc. Natl. Acad. Sci U.S.A. 109 (27), 1100211007

Evans, J.D., Lopez, D.L. (2004) Bacterial probiotics induce an immune response in the honey bee (Hymenoptera: Apidae). J. Econ. Entomol. 97 (3), 752-756

Forsgren, E., Olofsson, T.C., Vasquez, A., Fries, I. (2010) Novel lactic acid bacteria inhibiting Paenibacillus larvae in honey bee larvae. Apidologie 41 (1), 99-108

Gavriel, S., Jurkevitch, E., Gazit, Y., Yuval, B. (2011) Bacterially enriched diet improves sexual performance of sterile male Mediterranean fruit flies. J. Appl. Entomol. 135 (7), 564-573

Gilliam, M., Buchmann, S.L., Lorenz, B.J., Roubik, D.W. (1985) Microbiology of the larval provisions of the stingless bee, Trigona hypogea, an obligate necrophage. Biotropica 17 (1), 28-31

Gilliam, M., Buchmann, S.L., Lorenz, B.J., Schmalzel, R.J. (1990) Bacteria belonging to the genus Bacillus associated with three species of solitary bees. Apidologie 21 (2), 99-105

Hoover, S.E.R., Higo, H.A., Winston, M.L. (2006) Worker honey bee ovary development: Seasonal variation and the influence of larval and adult nutrition. J. Comp. Physiol. B. 176 (1), 55-63

Kawakami, S.-I., Yamada, T., Nakanishi, N., Cai, Y.M. (2010) Effect of probiotics on bacterial flora of various gastrointestinal regions in Holstein calves. J. Anim. Vet. Adv. 9 (11), 1556-1559

Kazimierczak-Baryczko, M., Szymas, B. (2006) Improvement of the composition of pollen substitute for honey bee (Apis mellifera L.), through implementation of probiotic preparations. J. Apic Sci 50 (1), 15-23

Kaznowski, A., Szymas, B., Jazdzinska, E., Kazimierczak, M., Paetz, H., Mokracka, J. (2005) The effects of probiotic supplementation on the content of intestinal microflora and chemical composition of worker honey bees (Apis mellifera). J. Apic. Res. 44(1), 10-14 
Koch, H., Schmid-Hempel, P. (2011) Socially transmitted gut microbiota protect bumble bees against an intestinal parasite. Proc. Natl. Acad. Sci U.S.A. 108(48), 19288-19292

Koch, H., Schmid-Hempel, P. (2012) Gut microbiota instead of host genotype drive the specificity in the interaction of a natural host-parasite system. Ecol. Lett. 15(10), 1095-1103

Kwong, W.K., Engel, P., Koch, H., Moran, N.A. (2014) Genomics and host specialization of honey bee and bumble bee gut symbionts. Proc. Natl. Acad. Sci U.S.A. 111 (31), 11509-11514

Losey, J.E., Vaughan, M. (2006) The economic value of ecological services provided by insects. BioSci. 56 (4), 311-323

Maggi, M., Negri, P., Plischuk, S., Szawarski, N., De Piano, F., De Feudis, L., Eguaras, M., Audisio, C. (2013) Effects of the organic acids produced by a lactic acid bacterium in Apis mellifera colony development, Nosema ceranae control and fumagillin efficiency. Vet. Microbiol. 167 (3-4), 474-483

Magnadottir, B. (2010) Immunological control of fish diseases. Mar. Biotechnol. (NY) 12 (4), 361-379

Martinson, V.G., Danforth, B.N., Minckley, R.L., Rueppell, O., Tingek, S., Moran, N.A. (2011) A simple and distinctive microbiota associated with honey bees and bumble bees. Mol. Ecol. 20 (3), 619-628

Masthan, K., Kumar, T.R., Rani, C.V.U., Murthy, C.V.N. (2010) Use of Lactobacillus acidophilus as a probiotics to improve cocoon production of mulberry silkworm, Bombyx mori (L.). J. Curr Sci 15(2), 445-449

Meeus, I., Mommaerts, V., Billiet, A., Mosallanejad, H., Van de Wiele, T., Wäckers, F., Smagghe, G. (2013) Assessment of mutualism between Bombus terrestris and its microbiota by use of microcolonies. Apidologie 44 (6), 708-719

Meeus, I., Parmentier, L., Billiet, A., Maebe, K., Van Nieuwerburgh, F., Deforce, D., Wäckers, F., Vandamme, P., Smagghe, G. (2015) 16S rRNA amplicon sequencing demonstrates that indoor-reared bumblebees (Bombus terrestris) harbor a core subset of bacteria normally associated with the wild host. PLoS One 10 (4), e 0125152

Moerman, R., Vanderplanck, M., Roger, N., Declèves, S., Wathelet, B., Rasmont, P., Fournier, D., Michez, D. (2016) Growth rate of bumblebee larvae is related to pollen amino acids. J. Econ. Entomol. 109 (1), 25-30

Niyazi, N., Lauzon, C.R., Shelly, T.E. (2004) Effect of probiotic adult diets on fitness components of sterile male Mediterranean fruit flies (Diptera: Tephritidae) under laboratory and field cage conditions. J. Econ. Entomol. 97 (5), 1570-1580

Oliver, K.M., Russell, J.A., Moran, N.A., Hunter, M.S. (2003) Facultative bacterial symbionts in aphids confer resistance to parasitic wasps. Proc. Natl. Acad. Sci U.S.A. 100 (4), 1803-1807

Oliver, K.M., Campos, J., Moran, N.A., Hunter, M.S. (2008) Population dynamics of defensive symbionts in aphids. Proc R Soc B Biol Sci. 275 (1632), 293-299
Olofsson, T.C., Vasquez, A. (2008) Detection and identification of a novel lactic acid bacterial flora within the honey stomach of the honeybee Apis mellifera. Curr. Microbiol. 57 (4), 356-363

Olofsson, T.C., Alsterfjord, M., Nilson, B., Butler, È., Vásquez, A. (2014) Lactobacillus apinorum sp. nov., Lactobacillus mellifer sp. nov., Lactobacillus mellis sp. nov., Lactobacillus melliventris sp. nov., Lactobacillus kimbladii sp. nov., Lactobacillus helsingborgensis sp. nov. and Lactobacillus kullabergensis sp. nov., isolated from the honey stomach of the honeybee Apis mellifera . Int. J. Syst. Evol Microbiol 64 (Pt 9), 3109-3119

Piccolo, G., Bovera, F., Lombardi, P., Mastellone, V., Nizza, S., Di Meo, C., Marono, S., Nizza, A. (2015) Effect of Lactobacillus plantarum on growth performance and hematological traits of European sea bass (Dicentrarchus labrax). Aquac. Int. 23 (4), 1025-1032

Roulston, T.H., Cane, J.H. (2000) Pollen nutritional content and digestibility for animals. Plant Syst. Evol. 222, 187-209

Sabree, Z.L., Hansen, A.K., Moran, N.A. (2012) Independent studies using deep sequencing resolve the same set of core bacterial species dominating gut communities of honey bees. PLoS One 7 (7), e41250

Sacchetti, P., Ghiardi, B., Granchietti, A., Stefanini, F.M., Belcari, A. (2014) Development of probiotic diets for the olive fly: evaluation of their effects on fly longevity and fecundity. Ann. Appl. Biol. 164 (1), 138-150

Schloss, P.D., Westcott, S.L., Ryabin, T., Hall, J.R., Hartmann, M., et al. (2009) Introducing mothur: open-source, platform-independent, communitysupported software for describing and comparing microbial communities. Appl. Environ. Microbiol. 75 (23), 7537-7541

Szymas, B., Langowska, A., Kazimierczak-Baryczko, M. (2012) Histological structure of the midgut of honey bees (Apis mellifera L.) fed pollen substitutes fortified with probiotics. J. Apic Sci 56(1), 5-12

Tian, B.Y., Fadhil, N.H., Powell, J.E., Kwong, W.K., Moran, N.A. (2012) Long-term exposure to antibiotics has caused accumulation of resistance determinants in the gut microbiota of honeybees. Microbiology 3 (6), e00377-00312

Vasquez, A., Forsgren, E., Fries, I., Paxton, R.J., Flaberg, E., Szekely, L., Olofsson, T.C. (2012) Symbionts as major modulators of insect health: Lactic acid bacteria and honeybees. PLoS One 7 (3), e33188

Velthuis, H.H.W., van Doorn, A. (2006) A century of advances in bumblebee domestication and the economic and environmental aspects of its commercialization for pollination. Apidologie 37 (4), 421-451

Vorburger, C., Gouskov, A. (2011) Only helpful when required: a longevity cost of harbouring defensive symbionts. J. Evol. Biol. 24 (7), 1611-1617

Wu, M.H., Sugimura, Y., Iwata, K., Takaya, N., Takamatsu, D., Kobayashi, M., Taylor, D., Kimura, K., Yoshiyama, M. (2014) Inhibitory effect of gut bacteria from the Japanese honey bee, Apis cerana japonica, against Melissococcus plutonius, the causal agent of European foulbrood disease. J. Insect Sci. 14, 129 